and particulars of whose cases $I$ tabulated in the paper already mentioned, I found that 6 per cent. were recorded to have had this symptom. Thus the difficulty is not to establish the existence of the hremorrhage, but to satisfactorily explain how it is produced. In the first cases which I observed, I was struck by the bounding pulse which preceded the bleeding, and thought that some increase of arterial tension might be an important agent, but a more careful investigation showed that, although the pulse was large and forcible, it was of decidedly low tension, a fact which was confirmed by sphygmograms taken before, during, and after the period when the patient was thoroughly under the influence of the drug. It seems, therefore, that we must fall back upon some chemical or physical change in the blood which makes it more readily transude through the capillaries, or else upon some secondary change in the walls of the vessels themselves. The point of practical importance to which I would call attention is this. Although I have carefully watched these cases for some years, I have never observed epistaxis or any other hæmorrhage occur until several hours, and generally not until some days, after the more common symptoms produced by too large a dose have been well marked. These symptoms are deafness, headache, vomiting, tinnitus aurium, and an irregularand slowpulse, this being the order of frequency with which they occurred in the series of 174 cases before referred to. It would therefore seem that if due regard were paid to these indications that the drug is beginning to produce its physiological effects, and an appropriate alteration made in the dose, the occurrence of loss of blood, which the patient can so ill afford, might be prevented. Patients differ very much in their susceptibility to these remedies, and the amount by which the dose must be reduced varies directly as the rapidity with which symptoms of poisoning are developed. A point requiring further investigation is whether the rather common practice of substituting salicine for salicylate of soda in identical doses, in cases in which the patient seems intolerant of the latter drug, is a thoroughly reliable proceeding. There is a general impression that salicine is less liable to produce ill effects than other preparations, but recorded cases do not fully bear this out. The chemists tell us that salicine is converted into salicylate of soda in the blood, and that, roughly, twenty grains of the original drug produce fifteen grains of the soda salt, and it is possible that any good effects which follow the substitution of one for the other might equally follow a corresponding diminution in the dose. In reference to this point, it is worth noting that in one of these cases, as well as in one of the fatal cases previously reported, salicine had been substituted for salicylate of soda two or more days before the hremorrhage occurred.

St. Thomas's-street, S.E.

\section{NOTES OF A}

\section{FATAL CASE OF PHENOL POISONING.}

BY A. G. R. FOULERTON, M.R.C.S., L.R.C.P., RESIDENT ASSIST.-SURGEON, ST. BARTHOLOMEW'S HOSPITAL, CHATHAM.

THE following case is thought worthy of publication, partly as being a fairly complete record of the poisonous action of carbolic acid, and partly because, as will be pointed out later on, the effect of the poison, as far as the stomach was concerned, differed in some respects from that described in one of our standard works on Forensic Medicine.

G. D-, aged thirty-six, a night watchman, was brought to St. Bartholomew's Hospital, Chatham, early in the morning of July 14th, in the following condition: Unconscious, collapsed, and smelling strongly of carbolic acid; face pale; pupils slightly dilated; conjunctival reflex absent; breatliing shallow and slightly stertorous; pulse weak; lips and interior of mouth of an appearance best described as dirty white. It was stated that, about twenty-five minutes previously, he ran from a place where he had been at work and told a fellow-workman that he had just swallowed some carbolic acid out of a bottle, thinking that it was cold tea. His speech, manner, and gait were then those of a drunken man. With assistance he was able to stagger to the hospital, a distance of rather more than a quarter of a mile. On arriving at the gate he made an ineffectual attempt to vomit, and immediately became unconscious. Some sulphate of zine was first given, and then a full dose of apomorphia was injected subcutaneously, in order to clear out the solid contents of the stomach preparatory to washing it out. As no vomiting occurred within a minute or two, and there was obviously no time to spare, the stomach-pump was at once used and the stomach well washed out with warm water, the portions first ejected being of milky appearance. After the stomach had been thoroughly washed out, it was seen that the man's general condition was rapidly becoming worse. Artificial respiration and subcutaneous injections of ether were then resorted to; without avail, however, for the patient died within twenty minutes of being brought to the hospital. The liquid swallowed was a crude preparation of a darkbrown colour, stated to contain 90 per cent. of pure carbolic acid, and used for disinfecting drains. The quantity swallowed was something less than three ounces.

Necropsy (made nine hours after death; day cool).Strong smell of carbolic acid noticeable about the body; slight hypostatic staining on the back and legs. Rigor mortis was present in a somewhat remarkable degree, it being with the greatest difficulty that the jaws could be forced open in order to inspect the mouth. There was a rather large stain of a light-brown colour at the right angle of the mouth. The oral mucous membrane presented the same appearance as that observed before death. Heart empty and very firmly contracted. Lungs normal. Liver much congested, and weighing $65 \mathrm{oz}$. Stomach slightly distended with some of the water used in the washing out, smelling strongly of carbolic acid; it contained no solid matter, and therefore, as no vomiting had occurred, had been empty at the time the accident happened. The peritoneal surface was much injected and mottled. The mucous membrane was of an ash-grey colour, apparently disorganised through its whole depth, corrugated and stiffened, with a faint pinkish staining between the rugæ here and there. These changes were more intensely marked at the cardiac than at the pyloric end. All over the surface of the mucous membrane were scattered small whitish elevations about the size of pin-heads, and easily detached from the subjacent surface. Examined under the microscope, they showed no definite structure, and were probably the result of the continued action of the acid upon minute blebs filled with coagulated serum. The duodenum was full of semi-digested matters, and in its first two inches or so presented slight traces of the action of the acid. The external coat of the cesophagus was congested; the attachment of the muscular to the nucous coat was loosened. The mucous lining was of a darker grey than that of the stomach, smooth, with an almost pearly lustre, but with well-marked longitudinal striation, similar to that sometimes produced by mineral acids. The brain and cord were not examined. Abdominal viscera not mentioned above were normal.

The first point to be noticed is as to the use of the stomach-pump in poisoning by corrosives. The general tendency of opinion is against its use in these circumstances, whilst the administration of emetics is allowed. But surely (unless, indeed, we presuppose an almost unpardonable display of clumsiness on the part of the operator) the vomiting following an enetic is not less likely to cause a rupture of the more or less disintegrated stomach than is the careful passage of an oiled flexible cesophageal tube to lead to its perforation. In practice, I believe, the best plan is first to give an emetic to evacuate the solid contents, which will greatly facilitate the next step, a good washing out with the stomach-punp. For, after all, a full stream of water is the quickest and most certain way of neutralising the poison. In exceptional cases, when odema of the pharynx has supervened, or when convulsions, which are not common with this class of poisons, are present, we possess an excellent alternative to the ordinary stomach. pump in the shape of a soft rubber tube with a funnel, used syphon-wise. Where there is obstruction, a tube of fine calibre may be used; and when strong convulsions are present, the greater flexibility and perfect softness of the rubber tube will prevent the patient from injuring himself. The explanation of the failure of the apomorphia in this case was found when the solution used was examined by daylight ; it was then seen that it had changed colour, and therefore presumably had decomposed.

A further fact to be noted in connexion with the case immediately under consideration was the state of the 
stomach and cesophagus. Drs. Guy and Ferrier ${ }^{1}$ state: "The mucous membranes of the mouth, gullet, and osophagus are pale, corrugated, sodden, and partially detached, and marked by local patches of hyperæemia or capillary hæmorrhage." "So also Dr. Stevenson," speaking of carbolic acid, says it "whitens and shrivels the membranes with which it comes in contact." Neither of these descriptions would fairly have described the state of things in this case. So far from being sodden, the lining of the stomach had a distinctly stiffened feeling, and its colour was a light grey, the cesophageal lining being considerably darker. The mucous membrane of the mouth could alone have been described as pale or white. These differences, however, might well be due to varying strength and quantity of the acid swallowed, and to a longer or shorter interval before death. Nor are they, indeed, of any practical importance, for the distinctive and enduring smell of the poison will by itself prevent a mistake as to its nature ever occurring. It may, perhaps, be worth while to add that the symptoms which presented themselves previously to the onset of insensibility, as narrated by an intelligent witness, bore a strong resemblance to those observed in a case of benzoline poisoning, published by me some little time ago, ${ }^{3}$ and justify to a certain extent the position that carbolic acid and benzine hold in Guy and Ferrier's classification, forming a subgroup of inebriant poisons. At the same time it would (in this case, at least) have been extremely hard to decide whether carbolic acid had greater claim to be considered as a neurotic or as a corrosive poison-an instance of the somewhat unsatisfactory nature, even from an empirical point of view, of the only classifications of poisons possible in our present state of knowledge.

\section{FOREIGN BODIES IN THE AIR PASSAGES.}

BY JOSEPH COLLIER, B.S., F.R.C.S.

ASSISTANT SURGEON TO THE HOSPITAL FOR SICEK CHILDREN, PENDLEBURY, MANCHESTER

THE entrance of a foreign body into the air passages, which in almost all cases takes place by inhalation, is an accident so serious, and calls for such prompt and accurate treatment, that it is well to have as many cases recorded as possible. This, perhaps, is additionally indicated, since the mishap is by no means cornmon, and since there would seem to be no two cases exactly similar either in symptoms or progress. All authorities are agreed as to the advisability of not being lulled into a false security by the apparent comfort of the patient, but urge the immediate performance of tracheotomy in every case where the foreign body is diagnosed as present below the larynx. But they are not equally agreed as to the virtue of any thorough examination of the upper air passages after the trachea has been opened, nor as to the extent to which search for and attempted removal by forceps should be carried. From an experience of several cases during the last few years, it is herein desired to urge the unwisdom of much meddling in the way of attempts at extraction by instruments, and as supporting evidence one case is detailed. After, but not before, free incision has been made into the trachea, a good inversion and succussion may be safely tried, and are especially likely to succeed if the foreign body is heavy and smooth but prolonged attempts with instruments may not only drive further down and fix more firmly the foreign body, but are almost certain to set up such an amount of irritation as will probably induce, especially in children, catarrhal pneumonia of fatal virulence.

W. $R$ - aged six years, was admitted into the Manchester Royal Infirmary on May 30th, 1886, under the care of $\mathrm{Mr}$. Whitehead. It was stated that the boy had been holding in his mouth a used percussion cap, when for some reason his mother liad struck him on the back. He seemed to have inspired deeply on receiving the blow, and then discovered that the cap was no longer in his mouth, nor could it be found. He immediately had several short but severe attacks of coughing, which subsided after about ten minutes

1 Principles of Forensic Medicine, 1880. 2 Quain's Dictionary of Medicine, art. Poisons. 3 The LanCet, Nov. 6th, 1886. and had not returned. On examination at the hospital he was breathing quietly, and the physical signs on both sides of the chest were apparently perfectly normal. He, however, complained of pain just above the sternum, and was continually grasping his throat with the right hand. From the uncertainty due to the absence of inoredefinitesymptoms, and to there being only the boy's statement as to the presence of the cap in his mouth, it was thought unjusti. fiable to perform immediate tracheotomy, but the patient was carefully watched, and preparation made to open the windpipe at once should urgent symptoms arise, or should the diagnosis of the foreign body being in the air passages become certain.

The patient slept well during the night, but next morning (May 31st), after a severe attack of coughing, examination showed that there was some obstruction to the entrance of air into the right lung. There was at first a peculiar shrill noise to be heard over the situation of the right bronchus, and the breath sounds were somewhat feeble over the same side. A few minutes later the shrill noise was no longerheard; the breath sounds on the right side were almost inaudible, movement almost imperceptible, and on per cussion that side was hyper-resonant. The presence of a foreign body seemed now to be certain, and it seemed equally certain that the body had entered the right bron. chus, at first partially and later almost completely obstruct. ing the air flow. While being prepared for tracheotomy a fit of coughing again came on, and on subsiding left the chest, as far as physical signs were concerned, once more normal.

Mr. Whitehead performed tracheotomy by the method described by him in THE LANCET of April 30th, 1887. An incision rather over an inch in length was made into the trachea, and through it a single futile attempt to feel the cap with a probe. No hard metallic substance, however, could be felt either in the trachea or the upper part of either bronchus. The wound in the trachea was kept open by passing a stout silver wire through the cartilage at the margin of the incision on each side, and fastening the wires with a tape behind the neck. In the coughing which followed the operation nothing was seen of the cap, and at the end of three days the silver wires had cut through the cartilage. In the absence of Gross's hooks or Golding Bird's dilator, a full-sized indiarubber tracheotomy tube was inserted for a portion of each day. A large aperture was made in the part of the tube looking towards the larynx, in order that breathing might be possible in at least one direction should the cap be coughed up.

The temperature rose slightly for a few days, and there was profuse, thin, sero-mucous expectoration for a similar. period. On June 14th, on removing the tube, an attack of coughing ensued, and by closing the throat wound and asking the boy to speak, it was found that the roice was. rough and sibilant. The laryngoscope showed a dark substance in the rima glottidis A Baker's indiarubber tube was replaced to prevent the body slipping back towards. the lungs, and in pqlling the tongue further forwards to obtain a better view of the larynx the body was released from between the vocal cords. As it was thus imprisoned in the upper part of the trachea, a pair of sinus forceps was inserted into the Baker's tube and expanded horizontally so as to block up the full lumen of the windpipe. The tube thus held was then inclined downwards in front and the percussion cap glided through the wound. It was a flattened copper cap, and was quite black. The boy made an uninterrupted recovery, the wound in the neck rapidly healed, and he left the hospital on June 24 th.

Manchester.

Devonshire Hospital, Buxton.--The report. submitted to the annual meeting of the trustees and sub. scribers held on the 6 th inst., sets forth that 2459 in-patients. had been received (seventy more than in the previous year). The number of out-patients was 215 . The general result of the medical treatment was very satisfactory. The financial position showed slightly increased receipts under various heads, one of which being an account of the patients under the powers of the governors of the Cotton Districts Convalescent Fund. The revenue had amounted to $\mathrm{f} 6660 \mathrm{ll}$ s. $4 \mathrm{~d}$., including $2120319 s .5 d$. from the Cotton Districts Convalescent Fund, and $£ 2745$ from annual subscriptions. The expenditure was $£ 602516 s .2 d$, and $£ 136 \quad 10 s$. had beet placed to the estate account. 